\title{
ON THE NONEXISTENCE OF A PROJECTION FROM FUNCTIONS OF $x$ TO FUNCTIONS OF $x^{n}$
}

\author{
P. MILMAN
}

\begin{abstract}
The subspace $\phi^{*} C^{\infty}\left(\mathbf{R}^{1}\right) \subset C^{\infty}\left(\mathbf{R}^{1}\right)$ of all $C^{\infty}$ functions of $\phi(x)=x^{n}, n=1,2,3, \ldots$, is a closed subspace of $C^{\infty}\left(\mathbf{R}^{1}\right)$ by Glaeser's Composition Theorem. We prove that for $n>2$ there does not exist a linear continuous projection $\pi$ from $C^{\infty}\left(\mathbf{R}^{1}\right)$ onto $\phi^{*} C^{\infty}\left(\mathbf{R}^{1}\right)$.
\end{abstract}

1. Introduction. Let $\phi: U \rightarrow V$, where $U$ and $V$ are open subsets of $\mathbf{R}^{n}$ and $\mathbf{R}^{m}, m \leqslant n$, respectively, be a semiproper real analytic mapping with a dense set of regular points; i.e. points $x \in U$ such that rank $d_{x} \phi=m$. Glaeser's Composition Theorem [2] provides the completeness of $\phi^{*} C^{\infty}(V)$ the pullback of $C^{\infty}$ functions on $V$.

Let $n=m=1, U$ and $V$ neighbourhoods of zero and $\phi(x)=x^{q}, q \geqslant 2$. The question arises whether there exists a linear continuous projection $\pi$ from $C^{\infty}(U)$ onto $\phi^{*} C^{\infty}(V)$. For $q=2$ the space $\phi^{*} C^{\infty}(V)$ coincides with the set of even functions [5] and the answer is positive (for example, $\pi$ is the projection along the subspace of odd functions). In this paper we show that the answer to the above question for $\phi(x)=x^{q}, q>2$, is negative; that is, we have the following.

TheOREM 1. For every $\phi(x)=x^{q}$, where $q>2$ is an integer, the subspace $\phi^{*} C^{\infty}(V)$ has no direct complementary subspace $\mathcal{C} \subset C^{\infty}(V)$; i.e., there does not exist a closed subspace $\mathcal{E} \subset C^{\infty}(U)$ such that

(1) $\mathcal{E} \cap \phi^{*} C^{\infty}(V)=\{0\}$;

(2) $\mathcal{E}+\phi^{*} C^{\infty}(V)=C^{\infty}(U)$.

The author would like to thank Edward Bierstone for statement of the question.

2. Notations. We consider two exact sequences of linear continuous mappings:

$$
\begin{aligned}
& 0 \rightarrow \mathscr{F} \quad \rightarrow \quad C^{\infty}(U) \quad \stackrel{T}{\rightarrow} F \quad F \quad \rightarrow \quad 0, \\
& 0 \rightarrow \underset{\mathscr{F}_{\text {even }}}{\rightarrow} C_{\text {even }}^{\infty}(U) \stackrel{I}{\rightarrow} F_{\text {even }} \rightarrow 0 \text {, }
\end{aligned}
$$

where $T$ is the canonical homomorphism associating to each $C^{\infty}$ function $f$ on $U$ its formal Taylor series $T f$ of infinite order at $0 \in U$. The kernel of

Received by the editors October 19, 1976.

AMS (MOS) subject classifications (1970). Primary 26A93, 26A96; Secondary 46A30.

Key words and phrases. Frechét space, Glaeser's Composition Theorem, open mapping theorem.

(1) American Mathematical Society 1977 
$T: C^{\infty}(U) \rightarrow F$ is the closed subspace $\mathscr{F}$ of all functions in $C^{\infty}(U)$ which are flat at 0 , and the factor ring $C^{\infty}(U) / \mathscr{F}$ is the linear topological space $F$ of formal power series. The spaces $\mathscr{F}_{\text {even }}, C_{\text {even }}^{\infty}(U)$ and $F_{\text {even }}$ are the closed subspaces of all even functions in $\mathscr{F}, C^{\infty}(U)$ and $F$ respectively.

3. Our result is a consequence of the following three lemmas.

LEMMA 1. Assume $\mathcal{E}$ is a closed subspace of $C^{\infty}(U)$ and is a complementary subspace to $\mathscr{F} \subset C^{\infty}(U)$; i.e.,

(1) $\mathscr{F} \cap \mathcal{E}=\{0\}$;

(2) algebraic sum $\mathscr{F}+\mathcal{E}$ is a closed subspace of $C^{\infty}(U)$.

Then $\operatorname{dim}_{\mathbf{R}} \mathcal{E}<\infty$.

LEMMA 2. Lemma 1 remains valid for spaces $C_{\text {even }}^{\infty}(U)$ and $\mathscr{F}_{\text {even }}$ substituted instead of $C^{\infty}(U)$ and $\mathscr{F}$ respectively.

LEMMA 3. Let $\phi(x)=x^{q}, q>2$. Then for odd $q, \mathscr{F} \subset \phi^{*} C^{\infty}(V)$ and for even $q, \mathscr{F}_{\text {even }} \subset \phi^{*} C^{\infty}(V)$.

We prove Lemmas 1,2 and 3 in $\S 4$.

Lemma 3 is also an immediate application of [4].

Proof of Theorem 1. Suppose the contrary for odd $q$ and let $\varepsilon$ be a direct complementary subspace to $\phi^{*} C^{\infty}(V)$. Then, using Lemma 3, we obtain the following diagram of linear continuous mappings

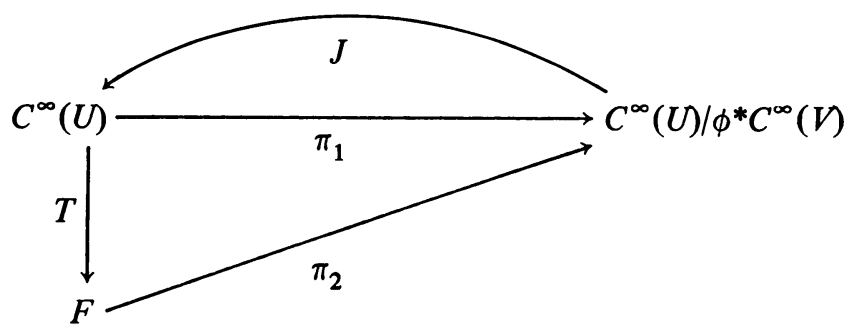

where $\pi_{1}$ is the canonical projection, $J: C^{\infty}(U) / \phi^{*} C^{\infty}(V) \rightarrow \mathcal{E}$ is a linear topological isomorphism and right inverse of $\pi_{1}$. The mapping $\pi_{2}$ is defined by $\pi_{2} \circ T=\pi_{1}$ because $\operatorname{ker} T=\mathscr{F} \subset \phi^{*} C^{\infty}(V)=\operatorname{ker} \pi_{1}$. The chain and product rules allow us to identify $\pi_{2}: F \rightarrow C^{\infty}(U) / \phi^{*} C^{\infty}(V)$ with the natural mapping $\pi_{3}: F \rightarrow F / \phi^{*} F$.

Therefore

$$
\operatorname{dim}_{\mathbf{R}} \mathcal{E}=\operatorname{dim}_{\mathbf{R}} C^{\infty}(U) / \phi^{*} C^{\infty}(V)=\operatorname{dim}_{\mathbf{R}} F / \phi^{*} F=\infty,
$$

$\mathscr{F} \cap \mathcal{E}=\{0\}$ and subspaces $T \mathscr{E}$ and $\mathscr{F}+\mathcal{E}=T^{-1}(T \mathscr{E})$ of $F$ and $C^{\infty}(U)$ respectively are closed. The latter contradicts Lemma 1 . Hence, Theorem 1 is proved for odd $q$.

Suppose the statement of Theorem 1 is not true for even $q$ and let $\varepsilon$ be a direct complementary subspace to $\phi^{*} C^{\infty}(V)$. Denote $\mathcal{E} \cap C_{\text {even }}^{\infty}(U)$ by $\mathcal{E}_{\text {even }}$. 
Since $\mathscr{F}_{\text {even }} \subset \phi^{*} C^{\infty}(V) \subset C_{\text {even }}^{\infty}(U) \subset C^{\infty}(U)$, the subspace $\varepsilon_{\text {even }}$ is a direct complementary subspace to $\phi^{*} C^{\infty}(V)$ in $C_{\text {even }}^{\infty}(U)$.

Repeating the proof for odd $q$, using the spaces $\mathscr{F}_{\text {even }}, C_{\text {even }}^{\infty}(U)$, $F_{\text {even }}$ and $\mathcal{E}_{\text {even }}$ instead of $\mathscr{F}, C^{\infty}(U), F$ and $\mathcal{E}$ respectively, we prove for even $q>2$

(1) $\operatorname{dim}_{\mathbf{R}} \varepsilon_{\text {even }}=\operatorname{dim}_{\mathbf{R}} C_{\text {even }}^{\infty}(U) / \phi^{*} C^{\infty}(V)=\operatorname{dim}_{\mathbf{R}} F_{\text {even }} / \phi^{*} F=\infty$;

(2) $\mathscr{F}_{\text {even }} \cap \mathcal{E}_{\text {even }}=\{0\}$;

(3) the subspaces $T \mathcal{E}_{\text {even }}$ and $\mathscr{F}_{\text {even }}+\mathcal{E}_{\text {even }}=T^{-1}\left(T \mathcal{E}_{\text {even }}\right)$ of $F_{\text {even }}$ and $C_{\text {even }}^{\infty}(U)$ respectively are closed.

The latter contradicts Lemma $2\left(\operatorname{dim}_{\mathbf{R}} \mathcal{E}_{\text {even }}<\infty\right)$. Hence, Theorem 1 is proved for all integers $q>2$.

4. In this section we prove Lemmas 1, 2 and 3.

Proof of Lemma 1. The mapping $T: \mathcal{E} \rightarrow F$ is a linear continuous mapping without kernel. Also $\mathscr{F}+\mathcal{E}$ and $T(\mathscr{F}+\mathcal{E})=T \mathcal{E}$ are closed subspaces of $C^{\infty}(U)$ and $F$ respectively. Using the open mapping theorem ([1, Chapter II, $\S 2]$ ) we obtain that $T: \mathcal{E} \rightarrow F$ is a linear topological isomorphism of $\mathcal{E}$ onto a closed subspace $T \mathcal{E}$ of $F$.

Now suppose $\operatorname{dim}_{\mathbf{R}} \mathcal{E}=\operatorname{dim}_{\mathbf{R}} T \mathcal{E}=\infty$. Every neighbourhood $W$ of zero in $F$ contains a closed subspace of $F$ of a finite codimension. Therefore, for every

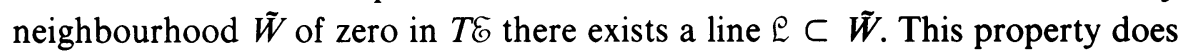
not take place for $\varepsilon$ considered in the induced topology from $C^{\infty}(U)$. Since $T: \mathcal{E} \rightarrow T \mathscr{E}$ is a linear topological isomorphism, we find that our assumption $\operatorname{dim}_{\mathbf{R}} \mathcal{E}=\infty$ is wrong.

Proof of Lemma 2. The proof of Lemma 2 repeats the proof of Lemma 1 after substituting the spaces $C_{\text {even }}^{\infty}(U), \mathscr{F}_{\text {even }}$ and $F_{\text {even }}$ for $C^{\infty}(U), \mathscr{F}$ and $F$ respectively.

Proof of Lemma 3. The proof uses arguments similar to the proof of Theorem 1 in [3]. Let us observe that $\mathscr{F} \subset \phi^{*} C^{0}(V)$ for odd $q$ and $\mathscr{F}_{\text {even }}$ $\subset \phi^{*} C^{0}(V)$ for even $q$. It remains to prove that $f(x)=g(\phi(x))$ and $f \in \mathscr{F}$ imply $g \in \mathscr{F} \subset C^{\infty}(V)$.

The mapping $\phi: U \rightarrow V$ is a diffeomorphism for $x \neq 0, x \in U$. Therefore $g$ is a $C^{\infty}$ function for $y \neq 0, y \in V$. Also, using the flatness of $f$ and

$$
f^{\prime}(x)=g^{\prime}(\phi(x)) \cdot \phi_{x}^{\prime}(x), \quad x \neq 0
$$

we obtain that $g^{\prime}(y)$ has a $C^{0}$ extension $g_{1}(y)$ equal to zero at $y=0$ such that $g_{1}(\phi(x))$ is a $C^{\infty}$ function (note $g_{1}(\phi(x))=g^{\prime}(\phi(x))$ for $x \neq 0$ ). The NewtonLeibniz formula

$$
\int_{a}^{b} u^{\prime}(t) d t=u(b)-u(a)
$$

implies now the existence of $g^{\prime}(0)$ and the equality $g^{\prime}(0)=\lim _{y \rightarrow 0} g^{\prime}(y)=0$. Further differentiating of

$$
f_{1}(x)=g_{1}(\phi(x)), \text { for all } x
$$


proves, using the above arguments for $g^{\prime}(y)$, that $\left(\left(d^{k} / d y^{k}\right) g\right)(y)$ has a $C^{0}$ extension $g_{k}(y)$. The function $g_{k}(y)$ is equal to zero at $y=0$ and $g_{k}(\phi(x))$ is a $C^{\infty}$ function. Therefore $\left(\left(d^{k} / d y^{k}\right) g\right)(0)$ exists and the equality

$$
\left(\frac{d^{k}}{d y^{k}} g\right)(0) \lim _{y \rightarrow 0}\left(\frac{d^{k}}{d y^{k}} g\right)(y)=0
$$

holds. Hence, $g \in \mathscr{F} \subset C^{\infty}(V)$ and $f \in \phi^{*} C^{\infty}(V)$. The lemma is proved.

\section{REFERENCES}

1. N. Dunford and J. T. Schwartz, Linear operators. I, General theory, Interscience, New York and London, 1958. MR 22 \#8302.

2. G. Glaeser, Fonctions composées différentiables, Ann. of Math. (2) 77 (1963), 193-209. MR 26 \#624.

3. P. Milman, The Malgrange-Mather division theorem (to appear).

4. J. C. Tougeron, An extension of Whitney's spectral theorem, Inst. Hautes Etudes Sci. Publ. Math. No. 40 (1971), 189-248.

5. H. Whitney, Differentiable even functions, Duke Math. J. 10 (1943), 159-160. MR 4, 193.

Department of Mathematics, University of Toronto, Toronto, Ontario, Canada M5S IAl 